\title{
Concentration of meteoritic free organic matter by fluid transport and adsorption
}

\author{
C. Potiszil ${ }^{1 *}$, R. Tanaka ${ }^{1}$, T. Ota' ${ }^{1}$, T. Kunihiro ${ }^{1}$, K. Kobayashi' ${ }^{1}$, E. Nakamura ${ }^{1}$
}

Abstract

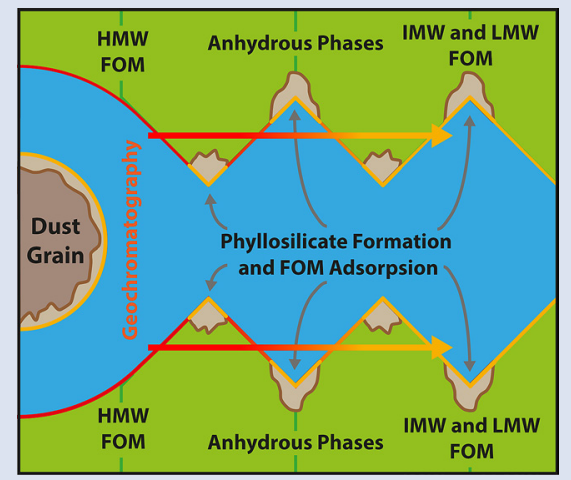

Carbonaceous chondrites contain many abiotic organic compounds, some of which are found in life on Earth. Both the mineral and organic matter phases, of these meteorites, have been affected by aqueous alteration processes. Whilst organic matter is known to be associated with phyllosilicate phases, no such relationship has yet been identified for specific organic compound classes. Furthermore, ongoing sample return missions, Hyabusa 2 and OSIRIS-Rex, are set to return potentially organic rich C-type asteroid samples to the Earth. Consequently, strategies to investigate organic-mineral relationships are required. Here we report spatial data for free/soluble organic matter (FOM/SOM) components (akylimidazole and alkylpyridine homologues) and mineral phases. Low and intermediate molecular weight alkylimidazole homologues are more widely distributed than higher molecular weight members, likely due to their affinity for the aqueous phase. On aqueous alteration of anhydrous mineral phases, transported FOM is adsorbed onto the surface or into the interlayers of the resulting phyllosilicates and thus concentrated and protected from oxidising fluids. Therefore, aiding the delivery of biologically relevant molecules to earth, shortly preceding the origin of life.

Received 26 June 2019 | Accepted 13 February 2020 | Published 17 March 2020

\section{Introduction}

CM2 carbonaceous chondrites, such as Murchison, demonstrate significant aqueous alteration recorded by their hydrous phases. Phyllosilicates replaced anhydrous minerals, such as olivine and pyroxene, originally accreted by the meteorite parent body/bodies (McSween, 1987; Brearley, 2006). Carbonaceous chondrites also contain abundant free and macromolecular organic matter (Pizzarello and Shock, 2010; Sephton, 2013) (FOM and MOM, respectively). Much of the meteoritic organic matter is thought to originate from within the interstellar medium (Sandford, 1996; Kerridge, 1999) and/or protosolar nebular (Ciesla and Sandford, 2012; Bekaert et al., 2018). Nevertheless, aqueous alteration has been indicated in the origin of some FOM compounds (Peltzer et al., 1984; Sephton et al., 1998). Subsequently, experiments have indicated the alteration of accreted organic matter could yield present day organic matter fractions and compounds within carbonaceous chondrites (Kebukawa et al., 2017; Vinogradoff et al., 2018).

Whilst many previous studies have examined the role of aqueous alteration on the mineral and organic phases, fewer have focussed on how aqueous alteration generates the relationships between these two phases. Phyllosilicates and carbonates are known to be associated with organic matter and are also known products of water-anhydrous mineral interaction. Although other interpretations exist, carbonates have been indicated as the products of organic matter oxidation (Alexander et al., 2015; Yesiltas and Kebukawa, 2016; Chan et al., 2017) and phyllosilicates are thought to protect or catalyse the synthesis/alteration of organic matter (Pearson et al., 2007; Kebukawa et al., 2010; Le Guillou et al., 2014; Yesiltas and Kebukawa, 2016).

Desorption electrospray ionisation-orbitrap-mass spectrometry (DESI-OT-MS) has been used previously to study Murchison (CM2) and Murray (CM2) chondrites (Hashiguchi and Naraoka, 2018; Naraoka and Hashiguchi, 2018). 2D DESIOT-MS maps of alkylimidazole, alkylpyridine and $\mathrm{C}_{n} \mathrm{H}_{2 n} \mathrm{NO}^{+}$ homologues were reported. It was found that for a given mass the distribution of the homologues was different and this was interpreted as a result of chromatographic separation through water-matrix interaction. Nevertheless, despite the use of scanning electron microscopy (SEM), no definitive relationship between mineral phases and the organic compounds could be established. The difference in the resolution of DESI-OT-MS $(\sim 50-200 \mu \mathrm{m})$ and SEM data (sub- $\mu \mathrm{m})$ was suggested as the likely reason for the lack of a mineral-organic relationship.

Raman spectroscopy can reveal the spatial distribution of both organic and mineral phases. Meteoritic organic matter was revealed to be a useful indicator of thermal alteration and was employed as a metamorphic thermometer (Bonal et al., 2006; Huss et al., 2006; Busemann et al., 2007). Previous Raman

1. Pheasant Memorial Laboratory, Institute for Planetary Materials, Okayama University, Yamada 827, Misasa, Tottori, 682-0193, Japan.
* Corresponding author (email: cpotiszil@okayama-u.ac.jp) 


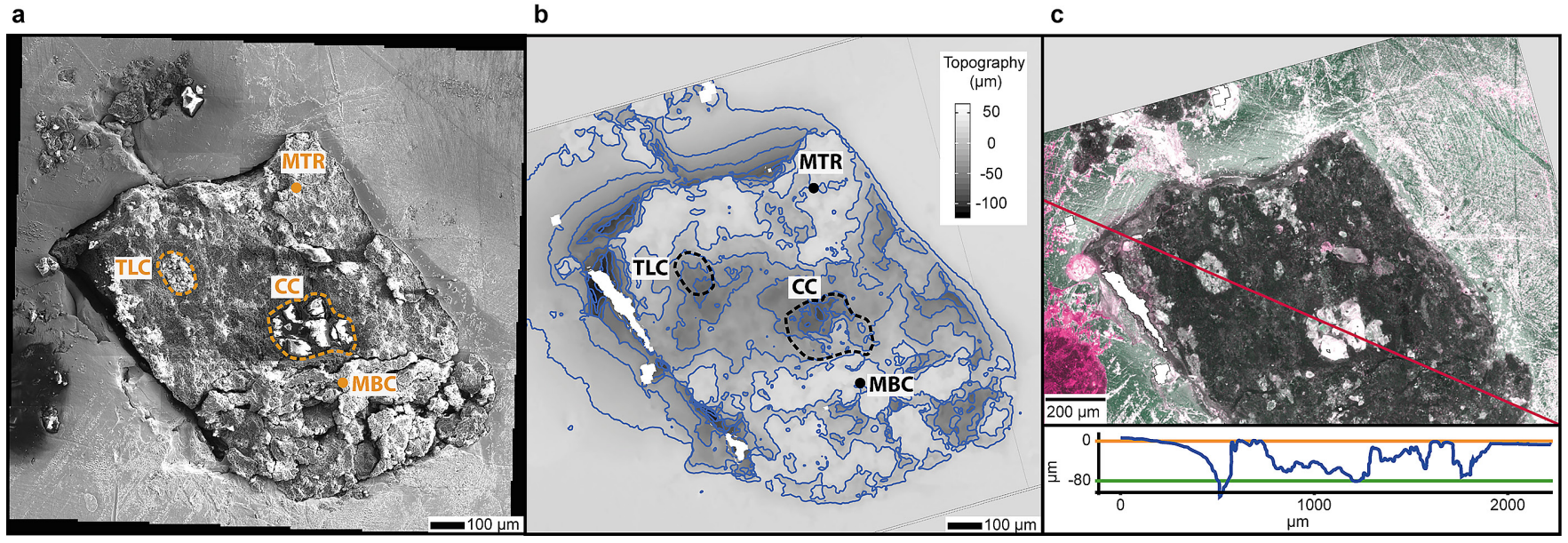

Figure 1 (a) An SEM image indicating the positions of chondrules (orange dashed lines) and matrix areas where EDX analyses were performed. (b) A topographic image of the sample surface obtained using a laser profiler. (c) An optical microscope image and topographic profile across the sample (red line).

spectroscopic studies have also identified olivine, pyroxene, Fe-oxides, Fe-oxyhydroxides and phyllosilicates in carbonaceous chondrites and enabled 2D mapping (El Amri et al., 2005; Kong and Wang, 2010; Wang et al., 2015). However, no previous studies have combined DESI-OT-MS and Raman mapping to probe carbonaceous chondrites.

\section{Results and Discussion}

In order to highlight relationships between FOM, mineral phases and textures, we imaged the sample using SEM. Two chondrules were identified from the SEM image (Fig. 1a), a large central chondrule (CC) and a smaller one to the top left (TLC). Energy dispersive X-ray (EDX) analysis indicates the $\mathrm{CC}$ is composed of forsteritic olivine (Fog9), whilst the TLC is a mixture of forsteritic olivine (Fo94) and low Ca pyroxene $\left(\mathrm{Wo}_{1} \mathrm{En}_{92}\right)$ (Table S-1). Considering the texture, mineral assemblages and chemical composition of the chondrules, the chondrules can be classified as type $1 \mathrm{PO}$ and POP, respectively (Scott and Krot, 2013). Despite significant aqueous alteration of CM2 chondrites on their parent body/bodies, the chondrules represent mostly unaltered mineral phases.

The matrix surrounding the TLC and matrix from the top right (MTR) and bottom centre (MBC) of the sample likely represent a mixture of phases. Nevertheless, if sulfur is not included then it is possible to explain the stoichiometry as being that of either serpentine or cronstedite, indicating a large phyllosilicate component is most likely present. The high phyllosilicate content of the matrix indicates that it was affected by aqueous alteration to a higher degree than the chondrules. The matrix has a higher porosity and consists of much smaller grain sizes than the chondrules. Therefore, the matrix is more permeable to, and reactive with, aqueous fluids than the chondrules (McSween, 1987; Brearley, 2006).

The DESI-OT-MS data for Murchison (CM2) records masses consistent with alkylimidazole and alkylpyridine homologues (Figs. 2, 3, S-1, S-2 and Table S-2), which have been previously reported (Hashiguchi and Naraoka, 2018; Naraoka and Hashiguchi, 2018). Analysis of a serpentinite blank using the same experimental set up and sample preparation confirmed that contamination was completely absent or not responsible for the spatial distribution of the compounds identified (Figs. S-3 and S-4).

To assess the contribution of topographic effects, a full assessment of the sample surface topography was undertaken using a laser profiler (Fig. 1b). High intensity areas are found in relation to both high and low topography in the DESI-OT-MS maps. Therefore, the observed intensity distributions cannot be explained by topography. Furthermore, Raman maps focussed on high and low topography (Figs. 2 and 3) were used to mitigate topographic effects, when comparing areas related to high adsorbed/interlayer water and DESI-OT-MS intensity. A more comprehensive and technical assessment of topography is available in the methods section.

Similar to previous studies (Hashiguchi and Naraoka, 2018; Naraoka and Hashiguchi, 2018), a disparity in the intensity distribution between low and intermediate and high molecular weight alkylimidazole homologues was observed (Fig. S-5). The correlation maps for low and intermediate molecular weight (LMW and IMW, respectively) alkylimidazoles (Fig. 2) record a widespread distribution of intermediate and high DESI/low Raman correlation, whereas the high molecular weight (HMW) maps show predominantly an intermediate/low Raman correlation. Such a relationship is compatible with a geochromatographic effect associated with the anhydrous unaltered phases. Thus, HMW homologues may have been adsorbed close to the accretion source, whilst IMW and LMW homologues were adsorbed further away.

The alkylpyridine homologues did not record a similar relationship, instead they show a relatively consistent intensity distribution between HMW, IMW and LMW homologues (Figs. 3 and S-6). It is possible that all homologues were deposited close to or at the site of accretion, potentially because they are less soluble or insoluble in aqueous solutions when compared to alkylimidazoles. Unfortunately, information concerning the solubility of the homologues studied here is very limited and standards were not available.

No studies have yet confirmed a relationship between specific FOM homologues and mineral phases such as phyllosilicates. Raman spectroscopy revealed an intense interlayer/ adsorbed water band between 3000-3800 $\mathrm{cm}^{-1}$ in Murchison. Both our Raman data (Fig. S-7) and those of previous studies link this water band to phyllosilicates (Kong and Wang, 2010; Wang et al., 2015). In the correlation maps for the alkylimidazoles (Fig. 2e-g.), there are almost no low/low, intermediate or high relationships observed for DESI/Raman. However, the LMW and IMW alkylimidazoles demonstrate widespread clusters of high/high and high/intermediate correlation for DESI/ Raman correlation maps. Therefore, where there are large abundances of phyllosilicates, there are either intermediate or high levels of the alkylimidazole homologues. Furthermore, there are more clusters of high/high and high/intermediate correlation within the LMW and IMW homologue correlation 
a). DESI (LMW)

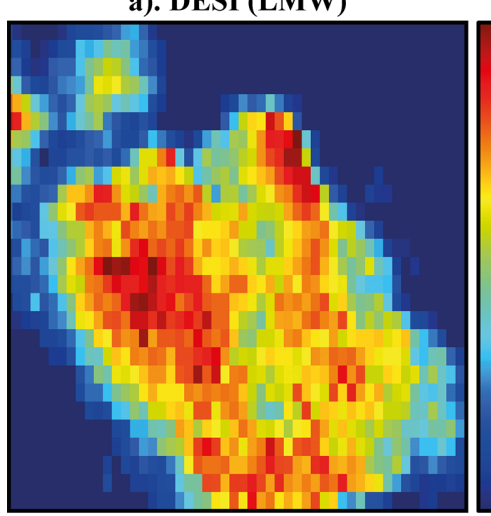

b). DESI (IMW)

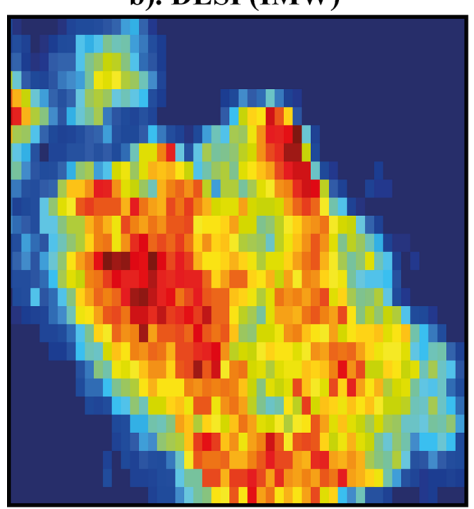

c). DESI (HMW)

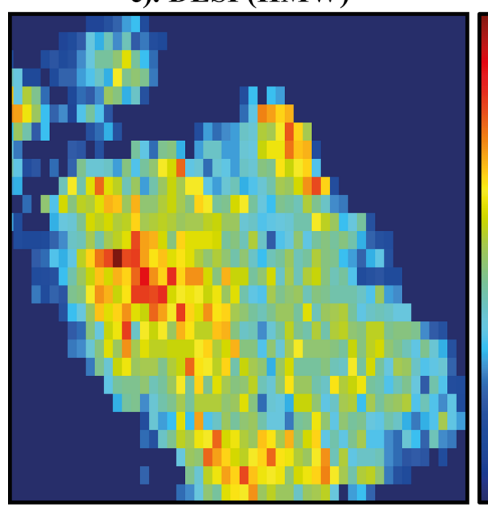

d). Correlation Map Key

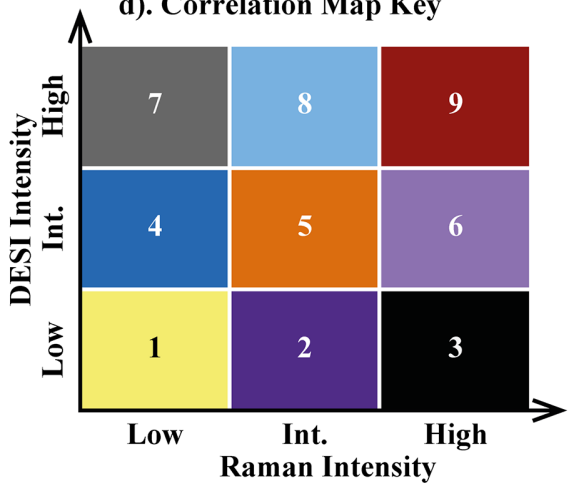

Low e). DESI (LMW)/Raman (HT)
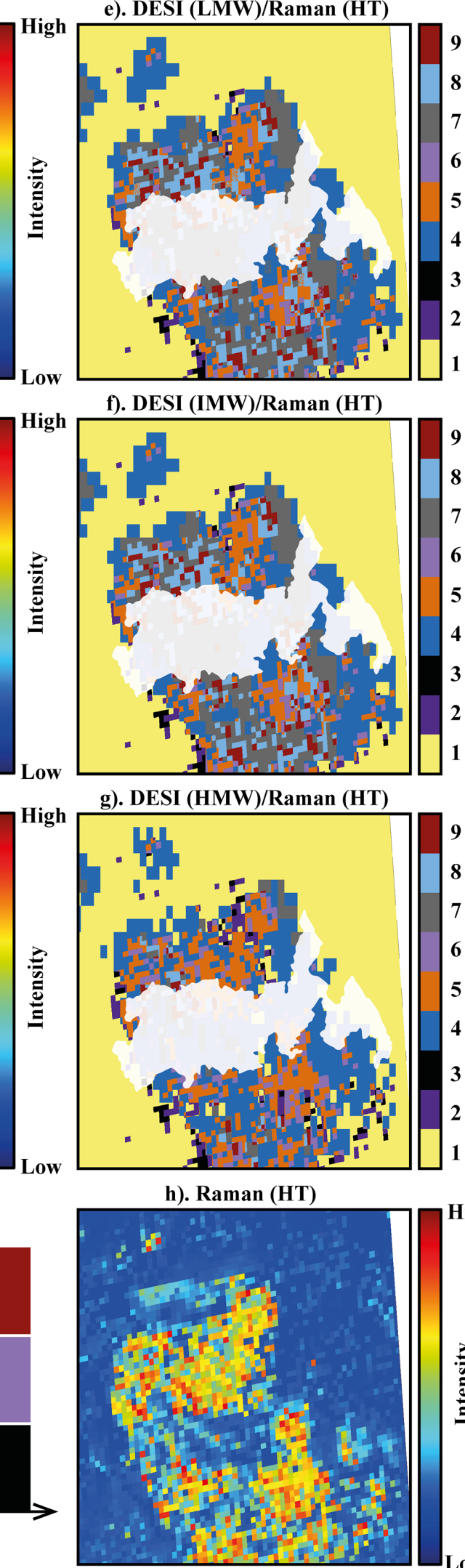

f). DESI (IMW)/Raman (HT)

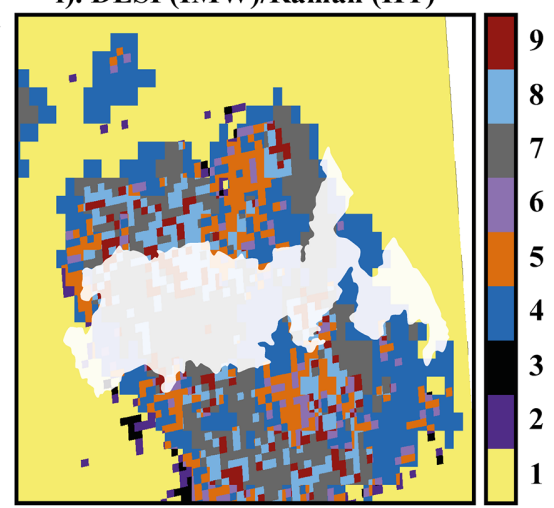

g). DESI (HMW)/Raman (HT)

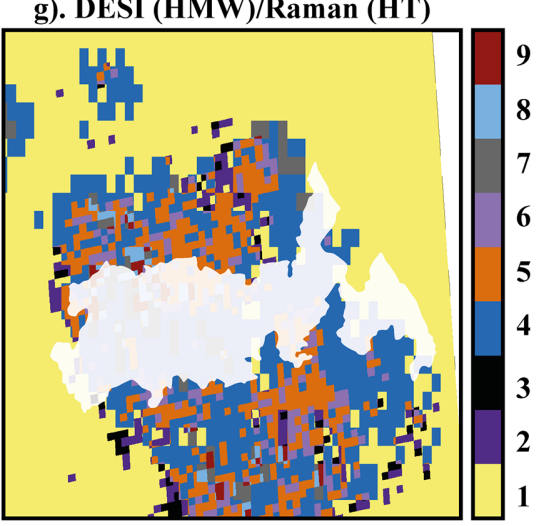

h). Raman (HT)

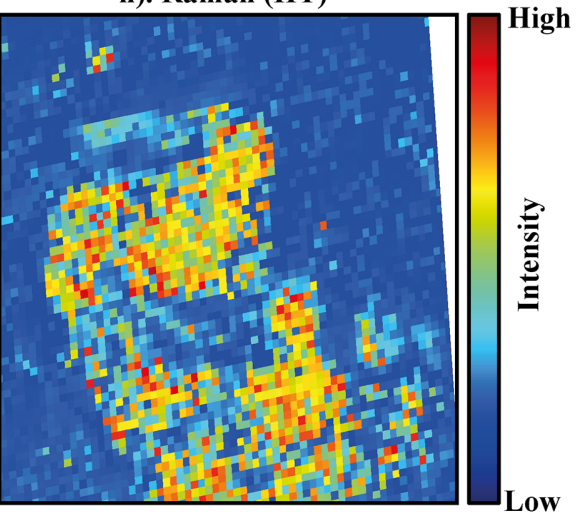

i). DESI (LMW)/Raman (LT)

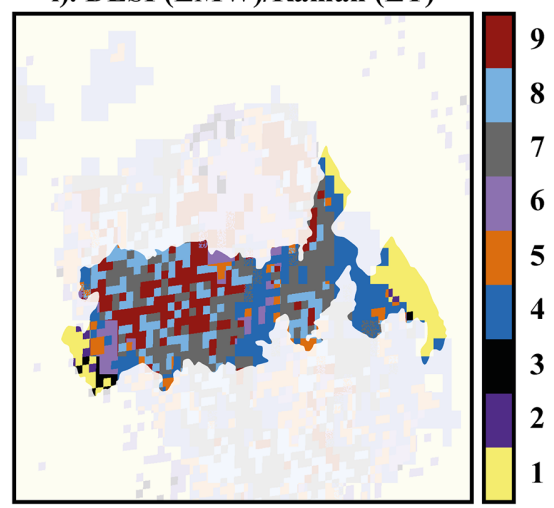

j). DESI (IMW)/Raman (LT)

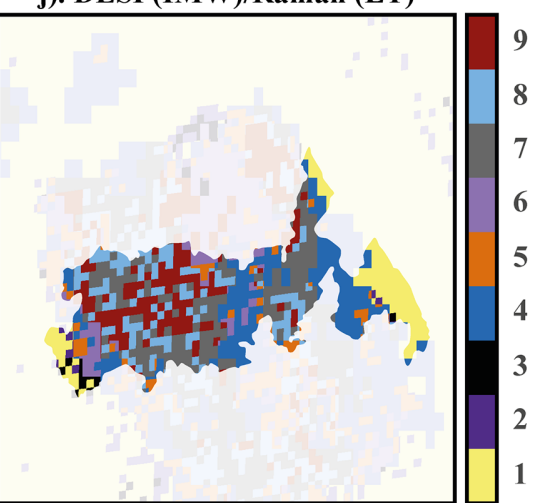

k). DESI (HMW)/Raman (LT)

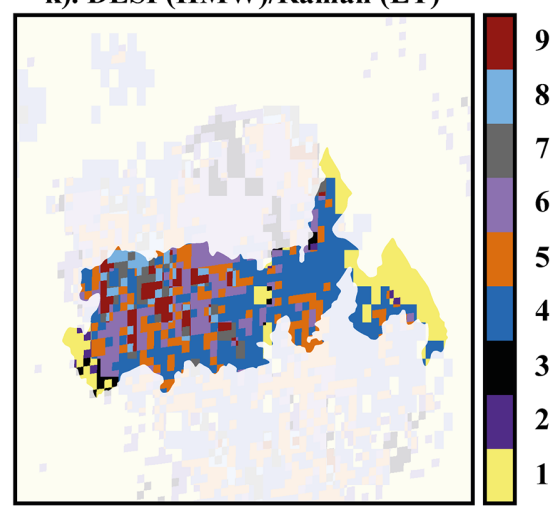

l). Raman (LT)

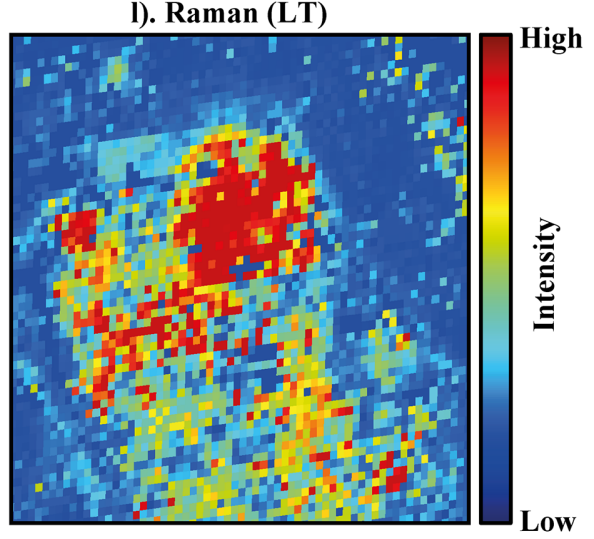

Figure 2 A compilation of DESI, Raman and correlation maps for selected alkylimidazole homologues. (a-c) DESI intensity maps for mass $139.12293,181.16978$ and 237.23225 , respectively. Highest normalised intensity is $8.79 \times 10^{-3}, 1.22 \times 10^{-2}$ and $3.84 \times 10^{-3}$, respectively. (d) A schematic outlining the criteria for the generation of DESI vs. Raman correlation maps. (e-g) Correlation maps showing the similarity between a given DESI and high topography (HT) Raman map. (h) A Raman intensity map focused on high topography, for the interlayer/adsorbed water band $\left(3000-3800 \mathrm{~cm}^{-1}\right)$. (i-k) Correlation maps showing the similarity between a given DESI and low topography (LT) Raman map. (I) As for h except focused on low topography. Note that for the correlation maps a partially transparent white mask has been used to highlight the high topography (top $40 \mu \mathrm{m}$ ) or low topography (below top $40 \mu \mathrm{m}$ ). 
a). DESI (LMW)

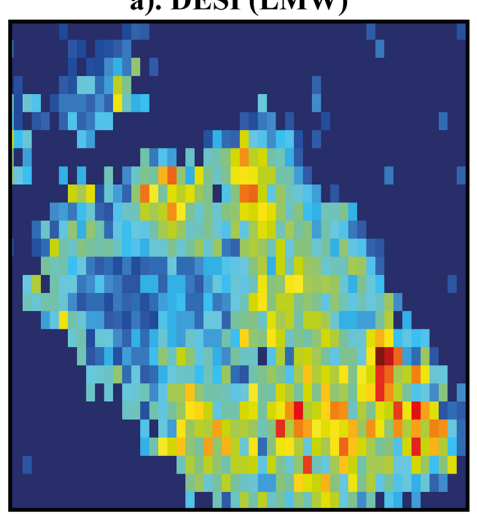

b). DESI (IMW)

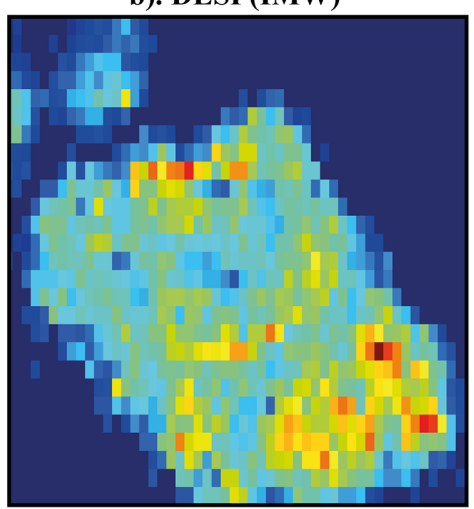

c). DESI (HMW)

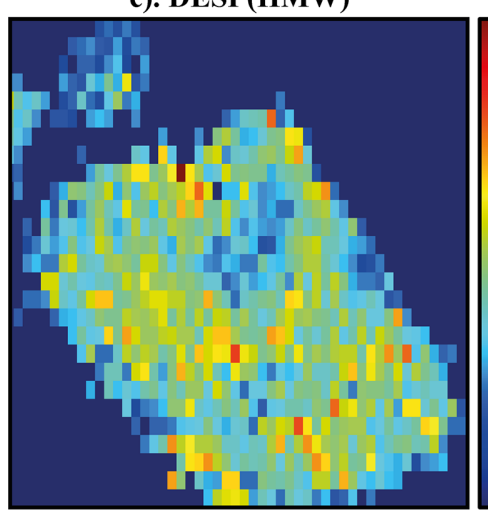

d). Correlation Map Key
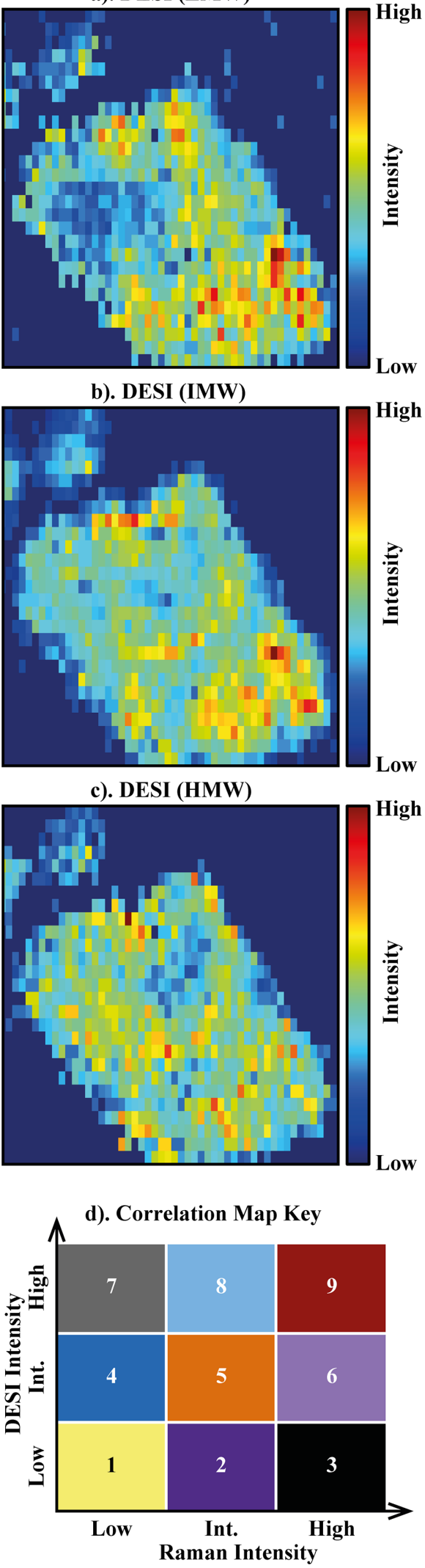

High

Low e). DESI (LMW)/Raman (HT)

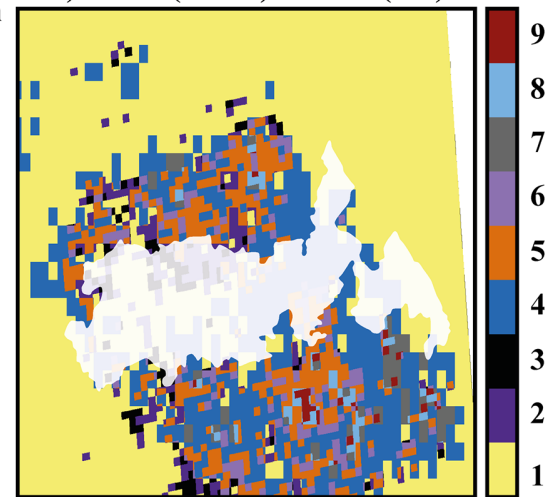

f). DESI (IMW)/Raman (HT)
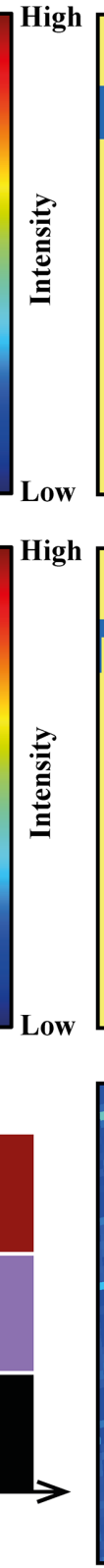

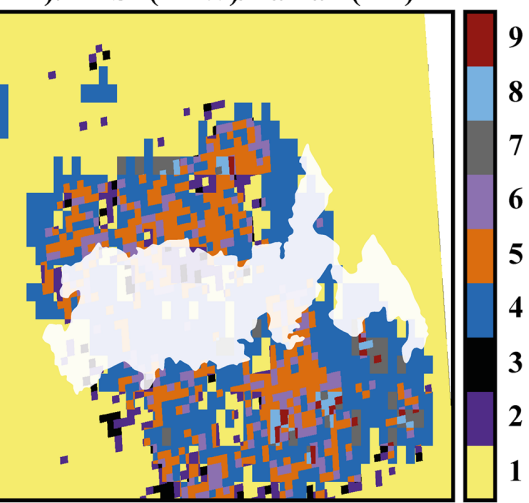

g). DESI (HMW)/Raman (HT)

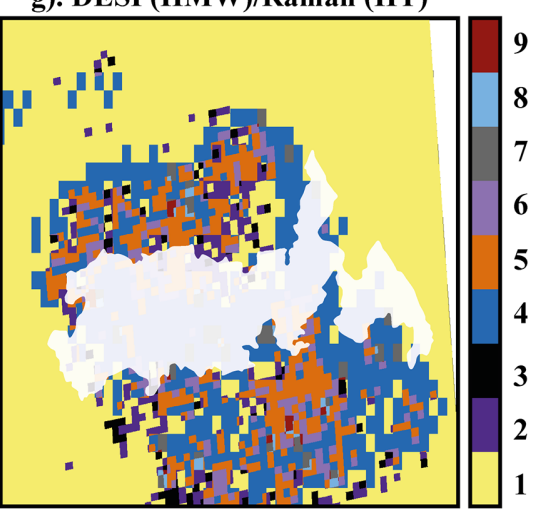

h). Raman (HT)

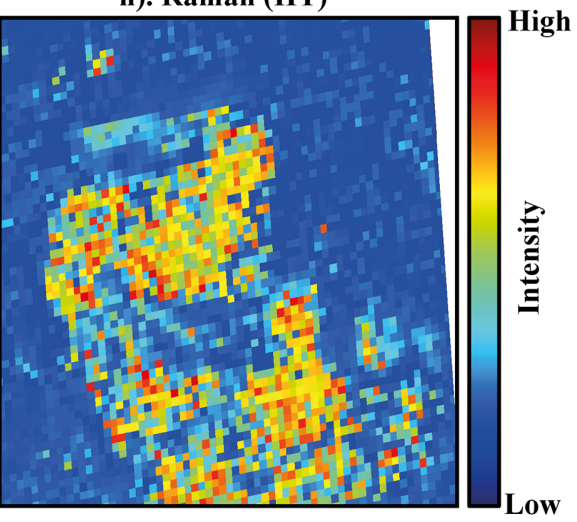

i). DESI (LMW)/Raman (LT)

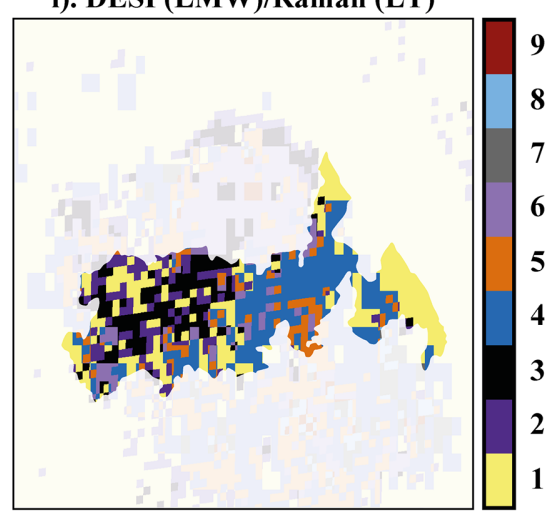

j). DESI (IMW)/Raman (LT)

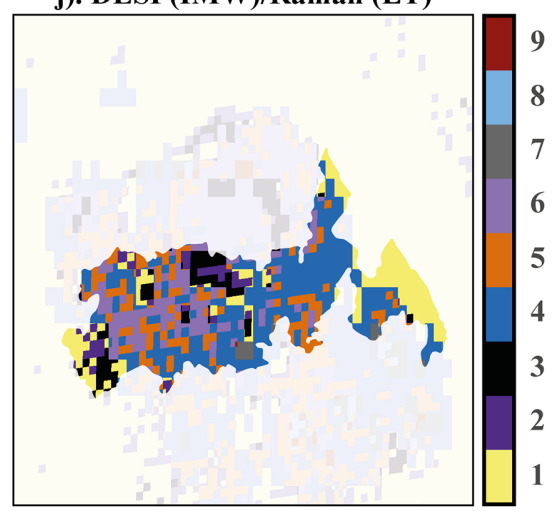

k). DESI (HMW)/Raman (LT)

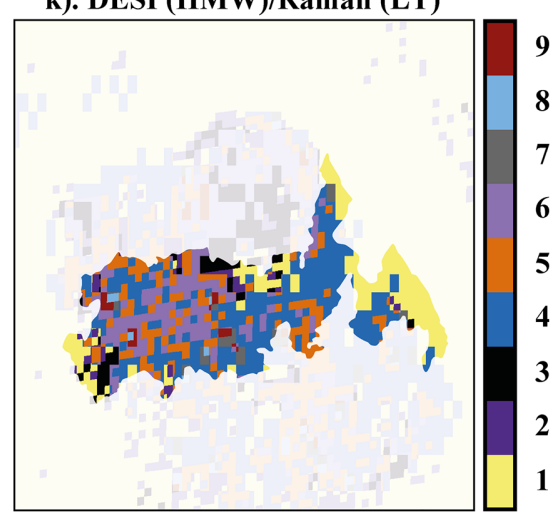

I). Raman (LT)

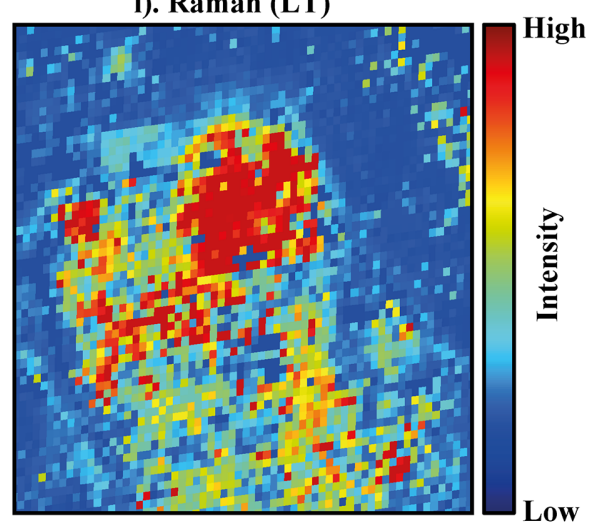

Figure 3 A compilation of DESI, Raman and correlation maps for selected alkylpyridine homologues. (a-c) DESI intensity maps for mass $136.11205,178.15891$ and 220.20580 , respectively. Highest normalised intensity is $2.17 \times 10^{-3}, 5.44 \times 10^{-2}$ and $2.58 \times 10^{-3}$, respectively. (d) A schematic outlining the criteria for the generation of DESI vs. Raman correlation maps. (e-g) Correlation maps showing the similarity between a given DESI and high topography (HT) Raman map. (h) A Raman intensity map focused on high topography, for the interlayer/adsorbed water band $\left(3000-3800 \mathrm{~cm}^{-1}\right)$. (i-k) Correlation maps showing the similarity between a given DESI and low topography (LT) Raman map. (I) As for h except focused on low topography. Note that for the correlation maps a partially transparent white mask has been used to highlight the high topography (top $40 \mu \mathrm{m}$ ) or low topography (below top $40 \mu \mathrm{m}$ ). 
a.

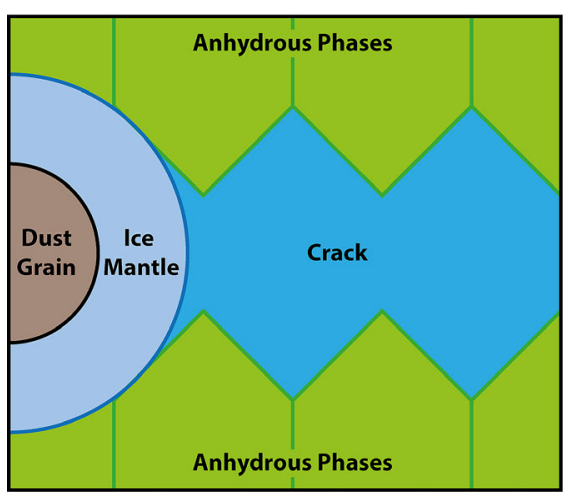

b.

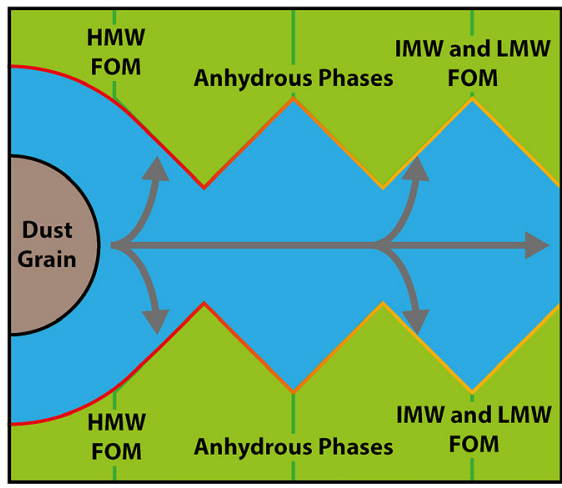

c.

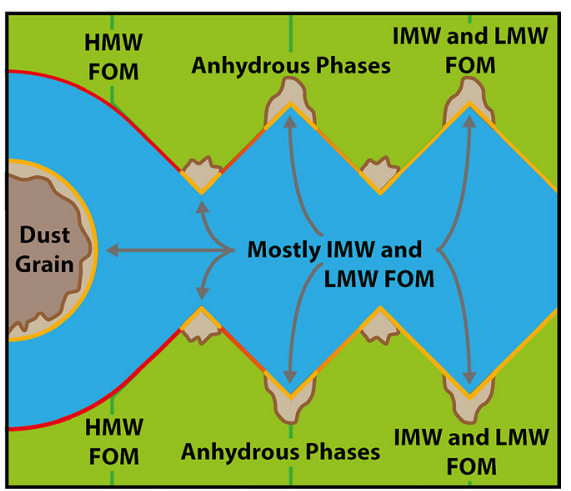

Figure 4 FOM distribution during aqueous alteration. (a) FOM and water ice are accreted by the parent body. (b) The ice melts and HMW FOM (red) adsorbs onto phases close to the site of accretion, but LMW and IMW FOM (light orange) are transported further into the matrix, generating a geochromatographic effect (red to light orange). (c) With the generation of phyllosilicates, adsorption of the remaining organic matter (LMW and IMW), as well as any previously adsorbed, occurs on these new charged mineral surfaces.

maps, compared to those for the HMW maps, indicating a stronger relationship between these homologues and phyllosilicates. Conversely, the alkylpyridine homologues show mainly an intermediate/low correlation for high topography DESI/Raman (Fig. 2e-g.), with clusters of intermediate/intermediate correlation. Furthermore, the distribution of the clusters is similar between each map, suggesting that a similar interaction with phyllosilicates occurred for the alkylpyridine homologues.

The results reported here suggest a more complex picture (Fig. 4) than those of previous studies (Hashiguchi and Naraoka, 2018; Naraoka and Hashiguchi, 2018). Alkylimidazole and alkylpyridine homologues contained within ice mantles could be introduced at the time of accretion. Aqueous fluids generated from accreted ice then mobilise the alkylimidazole homologues. HMW homologues are relatively hydrophobic due to their large chain lengths or numbers of substitutions (Naraoka et al., 2017) and may have thus been transported only a short distance (Fig. 4a.). Conversely, the LMW and IMW alkylimidazole homologues were transported further into the matrix of Murchison (Fig. 4b) and generated the spatial disparity between these homologues and their HMW counterparts. Subsequently, phyllosilicates form due to the alteration of anhydrous phases (Fig. 4c). Phyllosilicates have a larger surface area and surface charge, which allows the previously adsorbed homologues and LMW and IMW homologues in solution to be adsorbed on these phases. Such a process could give rise to the high/high and high/intermediate correlation clusters, from the LMW and IMW correlation maps of the alkylimidazole homologues. Indeed, organic adsorption onto phyllosilicate surfaces has been observed in marine clays (Kennedy et al., 2002) and has been proposed to protect organic matter from oxidising fluids on meteorite parent bodies (Yesiltas and Kebukawa, 2016).

In conclusion, aqueous alteration affects homologue series in different ways, which may be dependent on their affinity for aqueous rich fluids. For alkylimidazoles, aqueous alteration concentrates LMW and IMW FOM within and upon phyllosilicate phases. The organic compounds can then be protected from subsequent alteration and delivered to rocky planets over the course of the solar system's history. Many FOM components are important to living organisms, such as amino acids, sugars and nucleobases. The availability of these compounds early in the Earth's history could have aided the evolution of life.

\section{Acknowledgements}

We are greatly indebted to PML members for their assistance maintaining the laboratory. We would also like to thank Dr. Kengo Nojima, Tottori Institute of Industrial Technology, for providing the laser profiler information for the Murchison sample discussed within this manuscript. This work was supported by Ministry of Education, Culture and Sports, Science and Technology (MEXT) of Japan.

Editor: Liane G. Benning

\section{Additional Information}

Supplementary Information accompanies this letter at http:// www.geochemicalperspectivesletters.org/article2010.

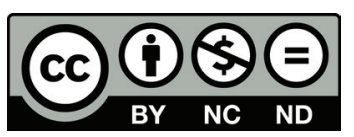

This work is distributed under the Creative Commons Attribution Non-Commercial No-Derivatives 4.0 License, which permits unrestricted distribution provided the original author and source are credited. The material may not be adapted (remixed, transformed or built upon) or used for commercial purposes without written permission from the author. Additional information is available at http://www.geochemicalperspectivesletters.org/ copyright-and-permissions.

Cite this letter as: Potiszil, C., Tanaka, R., Ota, T., Kunihiro, T., Kobayashi, K., Nakamura, E. (2020) Concentration of meteoritic free organic matter by fluid transport and adsorption. Geochem. Persp. Let. 13, 30-35.

\section{References}

Alexander, C.M.O., Bowden, R., Fogel, M.L., Howard, K.T. (2015) Carbonate abundances and isotopic compositions in chondrites. Meteoritics \& Planetary Science 50, 810-833.

Bekaert, D.V., Derenne, S., Tissandier, L., Marrocchi, Y., Anquetil, C., Marty, B., Charnoz, S. (2018) High-temperature Ionization-induced Synthesis of Biologically Relevant Molecules in the Protosolar Nebula. The Astrophysical Journal 859, 142.

Bonal, L., Quirico, E., Bourot-Denise, M., Montagnac, G. (2006) Determination of the petrologic type of CV3 chondrites by Raman spectroscopy of included organic matter. Geochimica et Cosmochimica Acta 70, 1849-1863.

BreARleY, A.J. (2006) The action of water. In: Lauretta, D.S., McSween Jr., H.Y. (Eds.) Meteorites and the Early Solar System II. University of Arizona Press, Tucson, 587-624. 
Busemann, H., Alexander, C.M.O., NitTler, L.R. (2007) Characterization of insoluble organic matter in primitive meteorites by microRaman spectroscopy. Meteoritics \& Planetary Science 42, 1387-1416.

Chan, Q.H.S., Zolensky, M.E., Bodnar, R.J., Farley, C., CheunG, J.C.H. (2017) Investigation of organo-carbonate associations in carbonaceous chondrites by Raman spectroscopy. Geochimica et Cosmochimica Acta 201, 392-409.

Ciesla, F.J., SANDFord, S.A. (2012) Organic Synthesis via Irradiation and Warming of Ice Grains in the Solar Nebula. Science 336, 452-454

El Amri, C., Maurel, M.-C., Sagon, G., Baron, M.-H. (2005) The micro-distribution of carbonaceous matter in the Murchison meteorite as investigated by Raman imaging. Spectrochimica Acta Part A: Molecular and Biomolecular Spectroscopy 61, 2049-2056.

HASHIgUCHI, M., NARAOKA, H. (2018) High-mass resolution molecular imaging of organic compounds on the surface of Murchison meteorite. Meteoritics \& Planetary Science 17, 1-17.

Huss, G.R., Rubin, A.E., Grossman, J.N. (2006) Thermal Metamorphism in Chondrites. In: Lauretta, D., McSween, H.Y. (Eds.) Meteorites and the early solar system II. University of Arizona Press, Tuscon, 567-586.

Kebukawa, Y., Nakashima, S., Ishikawa, M., Aizawa, K., Inoue, T., NaKamura-messenger, K., Zolensky, M.E. (2010) Spatial distribution of organic matter in the Bells CM2 chondrite using nearfield infrared microspectroscopy. Meteoritics \& Planetary Science 405, 394-405.

KebukaWa, Y., Chan, Q.H.S., Tachibana, S., Kobayashi, K., Zolensky, M.E. (2017) One-pot synthesis of amino acid precursors with insoluble organic matter in planetesimals with aqueous activity. Science Advances 3, e1602093.

Kennedy, M.J., Pevear, D.R., Hill, R.J. (2002) Mineral Surface Control of Organic Carbon in Black Shale. Science 295, 657-660.

KERRIDGE, J.F. (1999) Formation and processing of organics in the early solar system. Space Science Reviews 90, 275-288.

KonG, W.G., WANG, A. (2010) Planetary Laser Raman Spectroscopy for Surface Exploration on C/D-Type Asteroids - A Case Study. 41st Lunar and Planetary Science Conference, 2730-2731.

Le Guillou, C., Bernard, S., Brearley, A.J., Remusat, L. (2014) Evolution of organic matter in Orgueil, Murchison and Renazzo during parent body aqueous alteration: In situ investigations. Geochimica et Cosmochimica Acta 131, 368-392.

MCSwEEN, H.Y. (1987) Aqueous alteration in carbonaceous chondrites: Mass balance constraints on matrix mineralogy. Geochimica et Cosmochimica Acta 51, 2469-2477.

NARAOKA, H., HASHIGUCHI, M. (2018) In situ organic compound analysis on a meteorite surface by desorption electrospray ionization coupled with an Orbitrap mass spectrometer. Rapid Communications in Mass Spectrometry 32, 959-964.

Naraoka, H., Yamashita, Y., Yamaguchi, M., Orthous-Daunay, F.R. (2017) Molecular Evolution of N-Containing Cyclic Compounds in the Parent Body of the Murchison Meteorite. ACS Earth and Space Chemistry 1, 540-550.

Pearson, V.K., Kearsley, A.T., Sephton, M.A., Gilmour, I. (2007) The labelling of meteoritic organic material using osmium tetroxide vapour impregnation. Planetary and Space Science 55, 1310-1318.

Peltzer, E.T., BadA, J.L., Schlesinger, G., Miller, S.L. (1984) The chemical conditions on the parent body of the murchison meteorite: Some conclusions based on amino, hydroxy and dicarboxylic acids. Advances in Space Research 4, 69-74.

PizZARello, S., SHOCK, E. (2010) The organic composition of carbonaceous meteorites: the evolutionary story ahead of biochemistry. Cold Spring Harbor Perspectives in Biology 2, 1-19.

SANDFORD, S.A. (1996) The inventory of interstellar materials available for the formation of the solar system. Meteoritics and Planetary Science 31, 449-476.

ScotT, E.R.D., KROT, A.N. (2013) Chondrites and Their Components. In: Holland, H.D., Turekian, K.K. (Eds.) Treatise on Geochemistry: Second Edition. Elsevier, Oxford, 65-137.

SEPHTON, M.A. (2013) Organic Geochemistry of Meteorites. In: Holland, H.D., Turekian, K.K. (Eds.) Treatise on Geochemistry: Second Edition. Elsevier, Oxford, 1-31.

Sephton, M.A., Pillinger, C.T., Gilmour, I. (1998) 13C of free and macromolecular aromatic structures in the murchison meteorite. Geochimica et Cosmochimica Acta 62, 1821-1828

Vinogradoff, V., Bernard, S., Le Guillou, C., Remusat, L. (2018) Evolution of interstellar organic compounds under asteroidal hydrothermal conditions. Icarus 305, 358-370.

Wang, A., KoroteV, R.L., Jolliff, B.L., Ling, Z. (2015) Raman imaging of extraterrestrial materials. Planetary and Space Science 112, 23-34.
Yesiltas, M., KebukawA, Y. (2016) Associations of organic matter with minerals in Tagish Lake meteorite via high spatial resolution synchrotron-based FTIR microspectroscopy. Meteoritics and Planetary Science $51,584-595$. 